\title{
Guia de abordagem para orientação ao paciente para adesão à farmacoterapia na
}

\section{insuficiência cardíaca}

\author{
Approach guide for patient orientation for adherence to pharmacotherapy in heart failure \\ Guía de abordaje para la orientación del paciente sobre la adherencia a la farmacoterapia en la \\ insuficiencia cardíaca
}

\author{
Ranieri Carvalho Camuzi \\ ORCID: https://orcid.org/0000-0002-5584-8039 \\ Universidade Federal Fluminense, Brasil \\ E-mail: rcamuzi@id.uff.br \\ Jéssica Quintanilha Marcelo de Carvalho \\ ORCID: https://orcid.org/0000-0001-5617-4083 \\ Universidade Federal Fluminense, Brasil \\ E-mail: jessicaquintanilha@id.uff.br \\ Rafaela Tavares Peixoto \\ ORCID: https://orcid.org/0000-0002-9362-3770 \\ Ministério da Saúde, Brasil \\ E-mail: rafaelatpeixoto@gmail.com \\ Evani Leite de Freitas \\ ORCID: https://orcid.org/0000-0002-4622-6721 \\ Fundação Saúde/RJ, Brasil \\ E-mail: evanileite@id.uff.br \\ Evandro Tinoco Mesquita \\ ORCID: https://orcid.org/0000-0002-7452-3870 \\ Universidade Federal Fluminense, Brasil \\ E-mail: etmesquita@gmail.com \\ Selma Rodrigues de Castilho \\ ORCID: https://orcid.org/0000-0003-0272-4777 \\ Universidade Federal Fluminense, Brasil \\ E-mail: selmarc@id.uff.br
}

\begin{abstract}
Resumo
Objetivo: desenvolver um instrumento de consulta rápida e fácil para uniformizar a conduta de diferentes membros da equipe de atendimento para orientação farmacêutica e promoção da adesão à farmacoterapia na insuficiência cardíaca (IC). Método: estudo de desenvolvimento experimental que consistiu em reunir a experiência dos autores e recomendações divulgadas em publicações técnicas e científicas para elaboração de um instrumento para subsidiar a abordagem de orientação farmacêutica e promoção da adesão de pacientes com IC. Desenvolvimento: o Guia foi organizado na forma de um quadro que relaciona o tipo de abordagem de acordo com as respostas dos pacientes aos itens do Teste Morisky-Green (TMG), considerando os fatores intencionais e não intencionais que interferem no comportamento de adesão, conciliando a abordagem dos 4 itens do TMG à abordagem das 16 razões para não adesão de Culig. Acrescentou-se ainda um terceiro conjunto de fatores que são aqueles relacionados aos medicamentos. Conclusão: o Guia foi desenvolvido e aplicado, demonstrando-se uma ferramenta útil para agilizar e uniformizar as condutas durante o acompanhamento farmacoterapêutico a pacientes com IC, colaborando para melhores resultados nas intervenções para promoção da adesão.

Palavras-chave: Serviços de assistência farmacêutica; Adesão à medicação; Insuficiência cardíaca, Terapia; Uso de medicamentos; Serviços comunitários de farmácia.
\end{abstract}

\begin{abstract}
Objective: to develop a quick and easy consultation tool to standardize the conduct of different members of the care team for pharmaceutical guidance and promotion of adherence to pharmacotherapy in heart failure (HF). Method: experimental development study that consisted of bringing together the authors' experience and recommendations published in technical and scientific publications for the development of an instrument to support the pharmaceutical guidance approach and promotion of adherence of patients with HF. Development: the Guide was organized in the form of a table that lists the type of approach according to the patients' responses to the Morisky-Green Test (TMG) items, considering the intentional and unintentional factors that interfere with the adherence behavior, reconciling the approach of the 4 items of the TMG to the approach of 16 reasons for non-adherence by Culig. A third set of factors was also added, which are those related to medications. Conclusion: the Guide was developed and applied, proving to be a useful
\end{abstract}


tool to streamline and standardize the conduct during the pharmacotherapeutic follow-up to patients with HF, contributing to better results in interventions to promote adherence.

Keywords: Pharmaceutical services; Medication adherence; Heart failure, therapy; Drug utilization; Community pharmacy services.

\begin{abstract}
Resumen
Objetivo: desarrollar una herramienta de consulta rápida y sencilla para estandarizar la conducta de los diferentes miembros del equipo de atención para la orientación farmacéutica y promoción de la adherencia a la farmacoterapia en la insuficiencia cardíaca (IC). Método: estudio de desarrollo experimental que consistió en reunir la experiencia de los autores y las recomendaciones publicadas en publicaciones técnicas y científicas para el desarrollo de un instrumento de apoyo al enfoque de orientación farmacéutica y promoción de la adherencia de los pacientes con IC. Desarrollo: la Guía se organizó en forma de tabla que enumera el tipo de abordaje según las respuestas de los pacientes a los ítems del Test Morisky-Green (TMG), considerando los factores intencionales y no intencionales que interfieren con la conducta de adherencia, conciliando los aproximación de los 4 ítems de la TMG al abordaje de 16 motivos de no adherencia por parte de Culig. También se agregó un tercer conjunto de factores, que son los relacionados con los medicamentos. Conclusión: la Guía fue desarrollada y aplicada, resultando una herramienta útil para agilizar y estandarizar la conducta durante el seguimiento farmacoterapéutico de los pacientes con IC, contribuyendo a mejores resultados en las intervenciones para promover la adherencia.
\end{abstract}

Palabras clave: Servicios farmacéuticos; Cumplimiento de la medicación; Insuficiencia cardíaca/tratamiento; Utilización de medicamentos; Servicios comunitarios de farmacia.

\title{
1. Introdução
}

A insuficiência cardíaca (IC) é uma síndrome clínica complexa de caráter sistêmico. Corresponde a uma disfunção cardíaca que ocasiona inadequado suprimento sanguíneo para atender às necessidades metabólicas tissulares, na presença de retorno venoso normal, ou fazê-lo somente com elevadas pressões de enchimento, ocorrendo normalmente como consequência de enfermidades do próprio coração ou de outros órgãos (Rohde et al., 2018).

A IC é uma síndrome prevalente que, apesar dos avanços no tratamento, acarreta significativa morbidade e mortalidade, e se tornou um dos principais problemas de Saúde Pública. Atualmente, é a síndrome cuja incidência mais cresce entre as doenças cardiovasculares. São estimados 2 milhões de casos novos a cada ano com altos índices de mortalidade e prevalência, num total estimado de aproximadamente 23 milhões de pessoas acometidas pela síndrome no mundo (Bocchi et al., 2009; Jessup, 2014; Roger, 2013; Rohde et al., 2018).

Estudos têm enfatizado que a baixa adesão ao tratamento, tanto farmacológico como não farmacológico, representa um dos principais fatores precipitantes da descompensação da IC (Albuquerque et al., 2015; Barretto et al., 2008; Fitzgerald et al., 2011; Mangini et al., 2008; Marinker \& Shaw, 2003; van der Wal \& Jaarsma, 2008). O estudo BREATH identificou a falta de adesão como a principal causa para descompensação na IC em 1.263 pacientes com diagnóstico definitivo de IC, de diversas regiões do Brasil (Albuquerque et al., 2015).

Entendemos adesão como a medida em que o comportamento de uma pessoa, de tomar os medicamentos, seguir uma dieta e/ou mudar o estilo de vida, coincide com as recomendações de um profissional de saúde (Camuzi, 2017; Culig et al., 2011; Davis et al., 2014; WHO, 2003).

A baixa adesão às terapias de longo prazo compromete severamente a eficácia do tratamento tornando-se um problema crítico na saúde pública, tanto do ponto de vista da qualidade de vida, quanto da economia da saúde. As intervenções destinadas a melhorar a adesão proporcionariam retorno positivo significativo sobre o investimento por meio da prevenção primária (de fatores de risco) e de prevenção secundária (de resultados adversos para a saúde) (Culig et al., 2011).

Desta forma, a adesão é um modificador importante da eficácia do sistema de saúde (Culig et al., 2011). Aumentar a eficácia das intervenções de adesão pode ter impacto muito maior sobre a saúde da população do que qualquer melhoria nos tratamentos médicos específicos (Nieuwlaat et al., 2014).

Conforme abordado por Culig e colaboradores (2011), estudos têm encontrado significativa redução de custos e 
aumento na eficácia das intervenções de saúde que são atribuíveis a ações de baixo custo para melhorar a adesão. Sem um sistema que aborde os fatores determinantes da adesão, os avanços na tecnologia biomédica vão deixar de realizar o seu potencial para reduzir a carga das doenças crônicas. O acesso a medicamentos é necessário, mas não suficiente por si só para o sucesso do tratamento.

A literatura aponta que as intervenções farmacêuticas para identificar, prever e reduzir a falta de adesão terapêutica têm o potencial de melhorar os resultados clínicos em pacientes com IC. Da mesma forma, há evidências de que a inserção do farmacêutico na equipe multidisciplinar pode contribuir para melhorar a adesão à medicação e a adoção de estilos de vida mais saudáveis entre os pacientes com IC (Camuzi et al., 2021; Davis et al., 2014; Despaigne et al., 2012; Jackevicius et al., 2008).

Em 2003, a Organização Mundial da Saúde introduziu um modelo baseado em cinco dimensões para fatores que afetam a adesão. Nesse modelo, as categorias que afetam a adesão incluem: o paciente; a condição; o tratamento; os fatores socioeconômicos; e os fatores relacionados ao sistema de saúde (WHO, 2003). Nessa abordagem, as intervenções farmacêuticas se enquadram na categoria "tratamento", e estudos têm demonstrado que o conhecimento do paciente sobre os seus medicamentos e os esquemas posológicos é um dos principais fatores associados à boa adesão para pacientes de IC (Camuzi, 2017; Camuzi et al., 2021; Davis et al., 2014). E é nesse aspecto que se inserem as intervenções farmacêuticas para contribuir para melhorar o conhecimento do paciente sobre o seu tratamento, a posologia e a importância da adesão para alcançar melhores resultados terapêuticos e melhor qualidade de vida; afinal, a orientação sobre medicamentos está entre as atividades farmacêuticas mais básicas (López Cabezas et al., 2006).

Segundo uma revisão da literatura sobre o papel do farmacêutico na previsão e na melhoria da adesão à terapia medicamentosa em IC, entre as intervenções farmacêuticas descritas e mais efetivas estão a orientação farmacêutica em nível ambulatorial e/ou na alta hospitalar, ações educativas e o acompanhamento farmacoterapêutico (Davis et al., 2014).

A revisão de literatura realizada por Davis, Packard e Jackevicius (2014) constatou que, dentre os trabalhos que conseguiram melhores resultados na identificação de não adesão entre os pacientes de IC, estão aqueles que associam testes de medida de adesão pelas respostas dos pacientes acerca do comportamento de tomada dos medicamentos, como a abordagem do Teste Morisky-Green (TMG) (Morisky et al., 1986), a métodos baseados em dados administrativos sobre dispensação de medicamentos, tais como a razão de posse dos medicamentos (MPR) e a proporção de dias cobertos (PDC) (Karve et al., 2009). Davis, Packard e Jackevicius (2014) também consideram como vantagem do TMG a sua viabilidade para implementação na clínica cotidiana e o fato de ter sido validado e amplamente utilizado em doenças crônicas.

O TMG consiste em quatro questões, respondidas com "sim” ou "não" (Q1= Você, alguma vez, se esquece de tomar seus medicamentos? Q2=Você alguma vez se descuida ao tomar seus medicamentos? Q3= Quando você se sente melhor, você para de tomar seus medicamentos? Q4 = Quando você se sente mal (pior), você para de tomar os seus medicamentos?). Como resultado, considera-se com boa adesão apenas aqueles que respondem negativamente às quatro questões; no extremo oposto, estariam aqueles que respondem afirmativamente a todos os itens. Assim, é possível avaliar a atitude do paciente quanto à utilização de medicamentos, inferindo de forma indireta, a adesão ao tratamento (Camuzi et al., 2021; Morisky et al., 1986).

Além disso, pelas respostas ao TMG é possível classificar a atitude de não adesão em intencional ou não intencional, considerando-se que uma resposta afirmativa ao primeiro e/ou ao segundo item correspondem à não adesão não intencional, ou seja, o paciente tem a intenção de tomar o medicamento corretamente, mas deixa de fazê-lo involuntariamente; enquanto uma resposta afirmativa ao terceiro e/ou quarto item corresponde à não adesão intencional, ou seja, o paciente deixa de tomar o medicamento deliberadamente (Camuzi, 2017; Oliveira-Filho et al., 2014). Essa abordagem por tipo de comportamento de adesão (intencional e/ou não intencional) vem sendo cada vez mais empregada em estudos e a sua adoção se justifica também na prática cotidiana pela sua utilidade de auxiliar na escolha das possíveis e mais adequadas abordagens e intervenções para promoção da adesão terapêutica para cada tipo de paciente (Lowry et al., 2005; Náfrádi et al., 2016). 
Além do diagnóstico da não adesão, conhecer os fatores que contribuem para esse quadro é fundamental para se definirem as intervenções adequadas. Diversos trabalhos, desde os anos 1990, têm destacado a importância de perguntar aos pacientes sobre as suas crenças a respeito dos seus tratamento e medicamentos, utilizando-se de breves questionários, com destaque para o The Beliefs about Medicines Questionnaire (BMQ) (Horne et al., 1999) que foi validado de forma bem-sucedida com pacientes com asma, diabetes, doença renal crônica, doença psiquiátrica, doença cardiovascular e pacientes de clínica geral. Acreditando que essa estratégia (de perguntar aos pacientes sobre as suas crenças a respeito dos seus tratamento e medicamentos) pode ser adaptada a outras doenças crônicas que requerem adesão a tratamentos contínuos, Culig e colaboradores (2011) propuseram as 16 razões mais comuns para uma pessoa não aderir ao tratamento (Quadro 1).

Quadro 1 - As 16 razões para não utilizar os medicamentos conforme a prescrição.

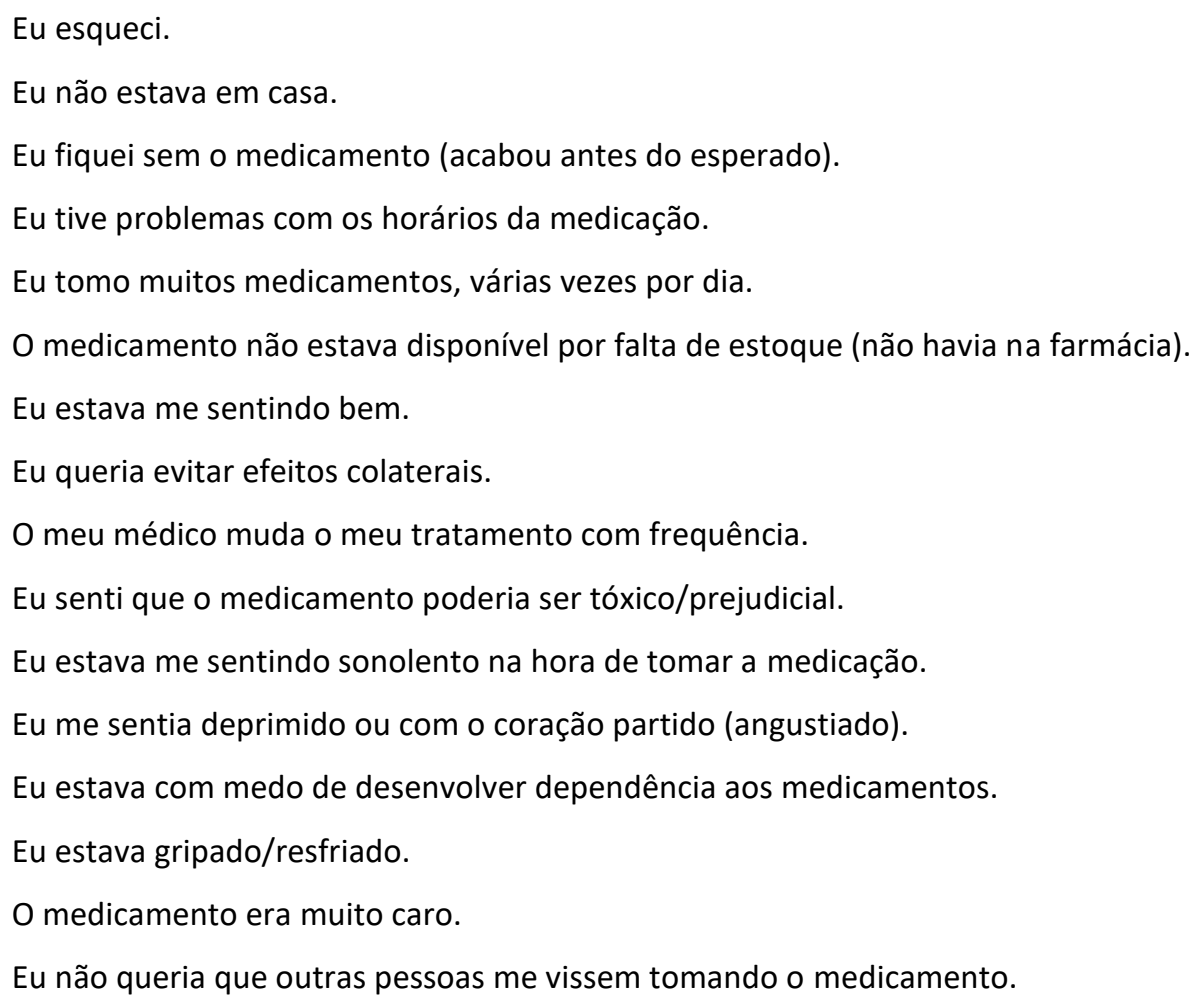

Fonte: Camuzi (2017).

Com base nesse contexto racional, durante o planejamento das rotinas para oferta de acompanhamento farmacoterapêutico a pacientes com insuficiência cardíaca em um centro clínico especializado, dentre os treinamentos necessários de prática clínica para os farmacêuticos da equipe, e visando a agilizar, uniformizar e garantir a precisão das informações na conduta da equipe, verificou-se a oportunidade de elaborar um instrumento de consulta para apoio à decisão quanto às possíveis intervenções para promoção da adesão conforme as necessidades e o perfil de cada paciente.

Diante da oportunidade percebida, estabeleceu-se como objetivo desenvolver um instrumento de consulta rápida e fácil para uniformizar a conduta de diferentes membros da equipe de atendimento para orientação farmacêutica e promoção da adesão à farmacoterapia na IC.

\section{Metodologia}

Trata-se de um estudo de desenvolvimento experimental (CNPq, 2021), que consistiu em reunir a experiência dos 
autores e recomendações divulgadas em publicações técnicas e científicas para elaboração de um instrumento para subsidiar a abordagem de orientação farmacêutica e promoção da adesão de pacientes com insuficiência cardíaca atendidos no âmbito do projeto "Implementação de Ações para Otimização da Assistência Farmacêutica a Pacientes com Insuficiência Cardíaca" (Camuzi, 2017).

O instrumento foi elaborado na forma de um guia de consulta rápida, partindo de um modelo apresentado por OliveiraFilho e colaboradores (2014), ao qual incorporaram-se a abordagem das razões para não adesão de Culig e colaboradores (2011) e os fatores relacionados ao medicamento que interferem na adesão (independentes do paciente), conforme proposto por Camuzi (2017).

Adicionalmente, o guia é composto por uma seção com informações relevantes para orientação farmacêutica sobre os 20 medicamentos mais frequentes nas prescrições dos pacientes com insuficiência cardíaca atendidos pelo projeto de pesquisa (Camuzi, 2017). Estas informações basearam-se no Formulário Terapêutico Nacional (Brasil, 2010), na Relação Nacional de Medicamentos Essenciais - RENAME (Brasil, 2015), no Bulário Eletrônico da Agência Nacional de Vigilância Sanitária (ANVISA) e no compêndio online Martindale - MICROMEDEX®.

O levantamento dos medicamentos mais prescritos foi realizado com base nas respostas dos pacientes à entrevista inicial, durante o período de recrutamento de pacientes, entre outubro de 2015 e março de 2016, para participarem do estudo autorizado pelo Comitê de Ética em Pesquisa da instituição - CAAE 43032415.9.3001.5272 (Camuzi, 2017).

\section{Desenvolvimento}

A demanda por um instrumento de consulta pelos farmacêuticos, para apoio à decisão quanto a intervenções necessárias e possíveis para cada caso, foi evidenciada durante a fase de recrutamento dos participantes, a partir dos dados levantados pelas entrevistas iniciais, quando se constatou baixa adesão para 3 em cada 4 pacientes (Camuzi, 2017).

Nosso grupo de pesquisa já acumulava boa experiência no atendimento a pacientes de insuficiência cardíaca (Camuzi et al., 2016, 2021), porém estava se preparando para iniciar o acompanhamento farmacoterapêutico em um novo cenário, centro especializado e referência nacional em cardiologia na rede pública de saúde, onde também foi realizado o treinamento das equipes que fizeram o recrutamento e o atendimento farmacêutico. Assim, criamos diversos instrumentos com vistas a garantir o melhor fluxo de atendimento, buscando conciliar otimização do tempo para realizar um bom atendimento, bem como adequados registros, avaliação e intervenções conforme as necessidades dos pacientes, adotando como foco principal a abordagem recomendada pelo Pharmacotherapy Workup (PW) (Morley et al., 2004).

No fluxo de atendimento dos participantes da pesquisa foram realizados: entrevista inicial (recrutamento), $1^{\mathrm{a}}$ consulta farmacêutica e $2^{\mathrm{a}}$ consulta farmacêutica para todos os participantes, sendo que mais consultas farmacêuticas foram necessárias para alguns pacientes.

A entrevista inicial tinha por objetivo coletar dados socioeconômicos, demográficos, clínicos e epidemiológicos, sobre a farmacoterapia, o comportamento de adesão, a razão para não utilização dos medicamentos, o nível de conhecimento do paciente quanto à prescrição de medicamentos, o índice de complexidade da farmacoterapia prescrita e a autoavaliação de saúde (Camuzi, 2017). Portanto, quando os participantes compareceram para a $1^{\text {a }}$ consulta, o farmacêutico que realizaria o seu atendimento já tinha estudado a sua situação por meio das informações previamente coletadas na entrevista inicial, a partir das quais identificava as suas necessidades e elaborava antecipadamente uma proposta de plano de cuidado individual. Porém, era preciso considerar a possibilidade de diferentes necessidades serem identificadas durante a consulta em si, o que nos incentivou a elaborar diversos instrumentos para consulta rápida, que pudessem subsidiar uma decisão segura e eficaz, durante o atendimento, quanto às intervenções mais adequadas em cada caso. Dentre esses instrumentos surgiu o "Guia de abordagem para orientação ao paciente para adesão à farmacoterapia na insuficiência cardíaca”, que foi originalmente desenvolvido pela 
nossa equipe.

O Guia (quadro 1) foi organizado na forma de um quadro que relaciona o tipo de abordagem de acordo com as respostas dos pacientes aos itens do Teste Morisky-Green (TMG), considerando os fatores intencionais e não intencionais que interferem no comportamento de adesão, conciliando a abordagem dos quatro itens de Morisky e colaboradores (1986), já trabalhada por Oliveira-Filho e colaboradores (2014), e a abordagem das 16 razões para não adesão de Culig e colaboradores (2011). Acrescentou-se ainda um terceiro conjunto de fatores que são aqueles relacionados aos medicamentos, o que harmoniza a abordagem final ao método Pharmacotherapy Workup (PW), no qual se enfatiza que a identificação dos problemas relacionados a medicamentos (PRM) deve considerar primeiro os fatores relacionados aos medicamentos, depois os fatores relacionados ao paciente (Morley et al., 2004).

O quadro é organizado em três seções. A primeira seção do instrumento é composta por linhas e colunas onde as informações são apresentadas de forma objetiva e correlacionadas conforme os fatores, os diagnósticos, as metas e os procedimentos recomendados. A consulta ao instrumento deve partir do diagnóstico de adesão, começando pela principal razão para não adesão, seguindo pela sua classificação quanto à natureza intencional ou não intencional, levando em consideração inclusive as respostas afirmativas do paciente a um ou mais itens do TMG. A segunda seção apresenta sucintamente as informações mais relevantes sobre as principais classes terapêuticas empregadas na farmacoterapia da IC. A terceira seção elenca as informações sobre cada medicamento, com foco em quatro aspectos: "vinculando o medicamento à programação do paciente"; "consequências da retirada do medicamento"; "causas potenciais para retirada"; e "alternativa para garantir provisão do medicamento". Cada seção será consultada conforme as necessidades identificadas para cada paciente.

Para melhor explicar a aplicação do Guia, considere os exemplos a seguir.

Exemplo 1: o paciente alega que frequentemente se esquece de tomar um ou mais medicamentos ("Eu esqueci”, dentre as razões para não utilizar os medicamentos); e respondeu SIM à primeira pergunta do TMG (Você, alguma vez, se esquece de tomar seus medicamentos?). Seguindo o Guia, verificamos que ambas as respostas estão previstas na coluna "Não Intencional"; e que a "Meta" prevista para esse caso (mesma coluna, linha inferior) é "Otimizar a memorização para tomada dos medicamentos"; e o "Procedimento" recomendado é "Fornecer um lembrete de fácil manuseio e identificar atividades diárias ou que o paciente faz regularmente por volta do(s) horário(s) em que deve tomar o(s) medicamentos(s) e explicar ao paciente que deve tomar os medicamentos nesse momento".

Exemplo 2: o paciente afirmou que algumas vezes se descuida do horário e toma o medicamento mais de meia hora atrasado, e geralmente isso acontece quando ele não está em casa (“Eu não estava em casa”, dentre as razões para não utilizar os medicamentos); e respondeu SIM à segunda pergunta do TMG (Você alguma vez se descuida ao tomar seus medicamentos?). Seguindo o Guia, verificamos que ambas as respostas estão previstas na coluna "Não Intencional"; e que a "Meta" prevista para esse caso (mesma coluna, linha inferior) é "Adaptar a posologia aos hábitos diários do paciente e abordar descuidos" ; e o "Procedimento" recomendado é "Fornecer um lembrete de fácil manuseio e identificar atividades diárias ou que o paciente faz regularmente por volta do(s) horário(s) em que deve tomar o(s) medicamentos(s) e explicar ao paciente que deve tomar os medicamentos nesse momento".

Exemplo 3: o paciente relatou que deixou de tomar o medicamento enalapril desde o fim de semana anterior porque estava de férias, se sentia bem e queria beber cerveja. Seguindo o Guia, verificamos que o comportamento relatado pelo paciente corresponde a "Eu estava me sentindo bem", dentre as razões para não utilizar os medicamentos; e à resposta SIM à terceira pergunta do TMG (Quando você se sente melhor, você para de tomar seus medicamentos?). Portanto, verificamos que ambas as respostas estão previstas na coluna "Intencional"; e que a "Meta" prevista para esse caso (mesma coluna, linha inferior) é “Evitar a interrupção do tratamento quando se sentir melhor"; e o "Procedimento" recomendado é "Explicar os princípios básicos da IC e seu tratamento para o paciente em termos que ele ou ela possa entender. Explicar como o medicamento funciona de uma 
forma simples e quais são as consequências ou os efeitos de cada medicamento se o paciente parar de tomá-lo". Considerando que o paciente afirmou que interrompeu o uso do medicamento enalapril, devemos verificar a seção "Informações sobre a classe terapêutica" e consultar as informações referentes ao próprio medicamento, onde, por exemplo, a coluna "Consequências da retirada do medicamento" informa que "A retirada abrupta desses medicamentos pode precipitar rebote hipertensivo em pacientes diabéticos com insuficiência renal crônica". Portanto, essas informações deverão ser abordadas na orientação ao paciente, principalmente se ele for diabético com insuficiência renal crônica, o que não é raro entre pacientes de IC, para conscientizá-lo quanto à importância de não suspender o uso do medicamento e evitar o consumo concomitante de bebida alcoólica.

Exemplo 4: o paciente afirma que, há duas semanas, não está usando o medicamento Cardilol@ prescrito pelo seu médico porque estava com pouco dinheiro e o medicamento está muito caro para ele. Seguindo o Guia, verificamos que o comportamento relatado corresponde a "O medicamento era muito caro", dentre as razões para não utilizar os medicamentos, porém sem correlação com as perguntas do TMG. Portanto, verificamos que o comportamento se enquadra na coluna "Fatores relacionados ao medicamento", e que a "Meta" deve ser "Adequar o esquema terapêutico e/ou auxiliar na facilitação do acesso aos medicamentos" e o "Procedimento" recomendado envolve "Sugerir ao prescritor: substituir por medicamentos disponíveis na rede pública de saúde, ou mais barato". Nesse caso, devemos verificar as informações referentes ao carvedilol, onde verificamos na coluna "Alternativa para garantir a provisão do medicamento" que o mesmo constava no elenco da Atenção Básica (AB), bem como era fornecido pela farmácia ambulatorial da instituição na época do estudo. Portanto, deveremos tentar contato com o prescritor sugerindo a prescrição de medicamento genérico ou similar, e recomendar ao paciente que busque o medicamento na farmácia ambulatorial da instituição ou que procure uma unidade básica de saúde.

Exemplo 5: o paciente informa que está inseguro em relação ao tratamento porque o seu médico muda com frequência a prescrição, que deixa de tomar os medicamentos quando não está se sentido bem, e que às vezes se descuida do horário e atrasa algumas doses. Dentre as razões para não utilizar os medicamentos, o comportamento relatado por este paciente corresponde a "O meu médico muda o meu tratamento com frequência", que está relacionado tanto na coluna "Intencional" quanto em "Fatores relacionados ao medicamento"; também corresponde a respostas afirmativas ao segundo e ao quarto itens do TMG ("Você alguma vez se descuida ao tomar seus medicamentos?" e "Algumas vezes, se você se sente pior, você para de tomar seus medicamentos?", respectivamente). Portanto, seguindo o Guia verificamos que a não adesão deste caso tem fatores não intencionais e intencionais, bem como fatores relacionados ao medicamento, demandando intervenções de diversas naturezas. Assim, considerando a resposta afirmativa ao segundo item do TMG, a "Meta" deve ser "Adaptar a posologia aos hábitos diários do paciente e abordar descuidos" e o "Procedimento" envolve "Fornecer um lembrete de fácil manuseio e identificar atividades diárias ou que o paciente faz regularmente por volta do(s) horário(s) em que deve tomar o(s) medicamentos(s) e explicar ao paciente que deve tomar os medicamentos nesse momento". Quanto à resposta afirmativa ao quarto item do TMG, a "Meta" é "Evitar a interrupção do tratamento quando se sentir pior", envolvendo o "Procedimento" "Ensinar ao paciente como monitorar os efeitos colaterais mais comuns do seu tratamento (taxas de abandono devido a efeitos colaterais devem ser investigadas). Apoiar e incentivar o paciente a relatar o problema ao médico". E, ainda, considerando a queixa do paciente quanto a mudanças frequentes de prescrição, verificando a coluna de "Fatores relacionados a medicamentos", a "Meta" será "Adequar o esquema terapêutico e/ou auxiliar na facilitação do acesso aos medicamentos", envolvendo o "Procedimento" “oferecer um 'feedback' ao prescritor quanto à insegurança do paciente com a frequência de alterações na sua prescrição". Cabe ressaltar que, nos casos em que a avaliação farmacêutica identifica mais de um PRM, mesmo que todos estejam relacionados à adesão, é recomendável considerar a possibilidade de priorização para intervir aos poucos, se possível, buscando resolver primeiro os PRM que possam ter consequências mais graves.

O Guia foi aplicado inicialmente durante o estudo de Camuzi (2017) em 73 consultas farmacêuticas, para um total de 39 pacientes, quando foram necessárias intervenções para resolver o PRM “Não adesão” em 44 ocasiões, no período de março a 
novembro de 2016. Os resultados demonstraram que houve mudança positiva no comportamento de não adesão; e verificou-se melhora estatisticamente significante do nível de conhecimento da prescrição pelo paciente (p-valor=0,006), da razão de posse dos medicamentos ( $p$-valor=0,026) e da autoavaliação de saúde ( $p$-valor=0,063), bem como houve aumento no número de pacientes que passaram a adquirir os seus medicamentos pelo programa Farmácia Popular (p-valor=0,016), acompanhado de redução do número de pacientes que precisavam comprar medicamentos (p-valor=0,070). Essas evidências sugerem impacto positivo do acompanhamento farmacoterapêutico com emprego do "Guia de abordagem para orientação ao paciente para adesão à farmacoterapia na insuficiência cardíaca”.

Consideramos como limitações do Guia: o fato de que ele foi desenvolvido e empregado a uma população limitada, necessitando estudos específicos para sua validação, com vistas a sua utilização mais ampla; bem como as informações sobre medicamentos precisarem ser atualizadas conforme o perfil de utilização/prescrição e atualizações nas respectivas monografias e bulas; e ainda em função da disponibilidade dos medicamentos nos pontos de acesso considerados para garantia da provisão do medicamento.

\section{Conclusão}

O "Guia de abordagem para orientação ao paciente para adesão à farmacoterapia na insuficiência cardíaca" consiste numa ferramenta de baixo custo, que disponibiliza informações e instruções aos farmacêuticos durante a consulta farmacêutica ou multidisciplinar, ou mesmo durante o estudo da situação do paciente pós-consulta, para acompanhamento farmacoterapêutico a pacientes com insuficiência cardíaca. Motivo pelo qual também tem grande potencial para aplicação no treinamento profissional para a prática do acompanhamento farmacoterapêutico, garantido uniformização de condutas e decisões acertadas para as intervenções necessárias.

Resultados da aplicação do Guia em uma população de IC verificaram mudanças positivas no comportamento de não adesão, bem como impacto positivo em outros fatores que também corroboram para melhorar a adesão terapêutica.

O Guia pode colaborar para melhores decisões clínicas na orientação de pacientes para promoção da adesão à farmacoterapia, sendo passível de atualização e de adaptação a diferentes públicos. 
Quadro 1 - Guia de abordagem para orientação ao paciente para adesão à farmacoterapia na insuficiência cardíaca.

\begin{tabular}{|c|c|c|c|c|c|}
\hline \multirow[b]{2}{*}{ Abordagem } & \multicolumn{4}{|c|}{ Fatores relacionados ao comportamento do PACIENTE } & \multirow{2}{*}{$\begin{array}{c}\text { Fatores relacionados ao } \\
\text { MEDICAMENTO }\end{array}$} \\
\hline & \multicolumn{2}{|c|}{ Não Intencional } & \multicolumn{2}{|c|}{ Intencional } & \\
\hline $\begin{array}{l}16 \text { razões de } \\
\text { Culig para não } \\
\text { utilizar os } \\
\text { medicamentos }\end{array}$ & \multicolumn{2}{|c|}{$\begin{array}{l}\text { 1. Eu esqueci. } \\
\text { 2. Eu não estava em casa. } \\
\text { 3. Eu fiquei sem o medicamento (acabou } \\
\text { antes do esperado). } \\
\text { 4. Eu tive problemas com os horários da } \\
\text { medicação. } \\
\text { 11. Eu estava me sentindo sonolento na hora } \\
\text { de tomar a medicação. } \\
\text { 12. Eu me sentia deprimido ou com o coração } \\
\text { partido (angustiado). } \\
\end{array}$} & \multicolumn{2}{|c|}{$\begin{array}{l}\text { 7. Eu estava me sentindo bem. } \\
\text { 8. Eu queria evitar efeitos colaterais. } \\
\text { 9. O meu médico muda o meu tratamento com frequência. } \\
\text { 10. Eu senti que o medicamento poderia ser tóxico/prejudicial. } \\
\text { 13. Eu estava com medo de desenvolver dependência aos } \\
\text { medicamentos. } \\
\text { 14. Eu estava gripado/resfriado. } \\
\text { 16. Eu não queria que outras pessoas me vissem tomando o } \\
\text { medicamento. }\end{array}$} & $\begin{array}{l}\text { 3. Eu fiquei sem o medicamento (acabou } \\
\text { antes do esperado). } \\
\text { 5. Eu tomo muitos medicamentos, várias } \\
\text { vezes por dia. } \\
\text { 6. O medicamento não estava disponível por } \\
\text { falta de estoque (não havia na farmácia). } \\
\text { 9. O meu médico muda o meu tratamento } \\
\text { com frequência. } \\
\text { 15. O medicamento era muito caro. }\end{array}$ \\
\hline Adesão (TMG) & $\begin{array}{l}\text { Você, alguma vez, } \\
\text { se esquece de tomar } \\
\text { seus medicamentos? }\end{array}$ & $\begin{array}{l}\text { Você alguma vez se } \\
\text { descuida ao tomar } \\
\text { seus medicamentos? }\end{array}$ & $\begin{array}{l}\text { Quando você se sente melhor, } \\
\text { você para de tomar seus } \\
\text { medicamentos? }\end{array}$ & $\begin{array}{c}\text { Algumas vezes, se você se sente } \\
\text { pior, você para de tomar seus } \\
\text { medicamentos? }\end{array}$ & - \\
\hline Meta & $\begin{array}{c}\text { Otimizar a } \\
\text { memorização para } \\
\text { tomada dos } \\
\text { medicamentos } \\
\end{array}$ & $\begin{array}{c}\text { Adaptar a posologia } \\
\text { aos hábitos diários do } \\
\text { paciente e abordar } \\
\text { descuidos } \\
\end{array}$ & $\begin{array}{l}\text { Evitar a interrupção do tratamento } \\
\text { quando se sentir melhor }\end{array}$ & $\begin{array}{l}\text { Evitar a interrupção do tratamento } \\
\text { quando se sentir pior }\end{array}$ & $\begin{array}{l}\text { Adequar o esquema terapêutico e/ou auxiliar } \\
\text { na facilitação do acesso aos medicamentos. }\end{array}$ \\
\hline Procedimento & \multicolumn{2}{|c|}{$\begin{array}{c}\text { Fornecer um lembrete de fácil manuseio e } \\
\text { identificar atividades diárias ou que o } \\
\text { paciente faz regularmente por volta do(s) } \\
\text { horário(s) em que deve tomar o(s) } \\
\text { medicamentos(s) e explicar ao paciente que } \\
\text { deve tomar os medicamentos nesse momento. }\end{array}$} & $\begin{array}{l}\text { Explicar os princípios básicos da } \\
\text { IC e seu tratamento para o } \\
\text { paciente em termos que ele ou ela } \\
\text { possa entender. Explicar como o } \\
\text { medicamento funciona de uma } \\
\text { forma simples e quais são as } \\
\text { consequências ou os efeitos de } \\
\text { cada medicamento se o paciente } \\
\text { parar de tomá-lo. }\end{array}$ & $\begin{array}{l}\text { Ensinar ao paciente como } \\
\text { monitorar os efeitos colaterais } \\
\text { mais comuns do seu tratamento } \\
\text { (taxas de abandono devido a } \\
\text { efeitos colaterais devem ser } \\
\text { investigadas). Apoiar e incentivar } \\
\text { o paciente a relatar o problema ao } \\
\text { médico. }\end{array}$ & $\begin{array}{l}\text { Sugerir ao prescritor: } \\
\text { - simplificar o esquema terapêutico; } \\
\text { - substituir por medicamentos disponíveis na } \\
\text { rede pública de saúde, ou mais barato; ou } \\
\text { - oferecer um 'feedback' ao prescritor quanto } \\
\text { à insegurança do paciente com a frequência } \\
\text { de alterações na sua prescrição. }\end{array}$ \\
\hline
\end{tabular}

Informações sobre a classe terapêutica

\begin{tabular}{|c|c|}
\hline $\begin{array}{l}\text { Agentes que atuam no sistema renina- } \\
\text { angiotensina, betabloqueadores e } \\
\text { bloqueadores dos canais de cálcio }\end{array}$ & $\begin{array}{c}\text { Esses medicamentos atuam dilatando os vasos sanguíneos e, em alguns casos, reduzindo a contratilidade do músculo cardíaco ou modulando o } \\
\text { ritmo do batimento cardíaco. Em geral, a sua ação depende de várias alterações hemodinâmicas, portanto pode demorar algumas semanas para que } \\
\text { os seus efeitos comecem a ser percebidos. }\end{array}$ \\
\hline $\begin{array}{l}\text { Antiagregantes plaquetários e } \\
\text { anticoagulantes }\end{array}$ & $\begin{array}{c}\text { O tratamento com estes medicamentos impede a formação de coágulos nos vasos sanguíneos. Ele pode ser usado para prevenir ou tratar infartos e } \\
\text { acidentes vasculares cerebrais }\end{array}$ \\
\hline Diuréticos & $\begin{array}{l}\text { Medicamentos dessa classe aumentam a perda de água através da inibição da reabsorção de íons sódio e cloreto nos rins. Ao tomar esses anti- } \\
\text { hipertensivos, haverá um aumento da frequência urinária, em grande parte devido ao aumento da perda de água. }\end{array}$ \\
\hline
\end{tabular}


Research, Society and Development, v. 10, n. 15, e142101522899, 2021

(CC BY 4.0) | ISSN 2525-3409 | DOI: http://dx.doi.org/10.33448/rsd-v10i15.22899

\begin{tabular}{|c|c|c|c|c|}
\hline & $\begin{array}{l}\text { Vinculando o medicamento à } \\
\text { programação do paciente }\end{array}$ & $\begin{array}{c}\text { Consequências da retirada } \\
\text { do medicamento }\end{array}$ & $\begin{array}{c}\text { Causas potenciais para } \\
\text { retirada }\end{array}$ & $\begin{array}{c}\text { Alternativa para garantir provisão do } \\
\text { MEDICAMENTO }\end{array}$ \\
\hline $\begin{array}{l}\text { ACIDO } \\
\text { ACETILSALICÍ- } \\
\text { LICO }\end{array}$ & $\begin{array}{l}\text { Horário ideal de tomada: na parte da manhã } \\
\text { e/ou à noite, com alimentos (administração } \\
\text { de aspirina à hora de dormir diminui a } \\
\text { elevação matinal da agregação plaquetária, } \\
\text { enquanto mantém a mesma eficácia } \\
\text { antiplaquetária durante as outras horas do dia, } \\
\text { em comparação com a administração desse } \\
\text { medicamento durante o dia) Não consumir } \\
\text { bebida alcoólica. }\end{array}$ & $\begin{array}{c}\text { A interrupção do tratamento com } \\
\text { ácido acetilsalicílico pode estar } \\
\text { associada com fatores de risco } \\
\text { cardiovasculares tradicionais e } \\
\text { trombose }\end{array}$ & $\begin{array}{l}\text { Efeitos colaterais gastrointestinais } \\
\text { (dor, dispepsia, indigestão), } \\
\text { sangramento }\end{array}$ & $\begin{array}{l}\mathrm{AB} \text { - comp. } 100 \text { e } 500 \mathrm{mg} \\
\mathrm{FP} \text { - comp. } 100 \text { e } 500 \mathrm{mg}\end{array}$ \\
\hline AMIODARONA & $\begin{array}{l}\text { Horário ideal de tomada: não encontrado. } \\
\text { Orientar que pode ser tomado com ou sem } \\
\text { alimento }\end{array}$ & $\begin{array}{l}\text { A descontinuação do tratamento } \\
\text { pode causar recorrência } \\
\text { imprevisível de arritmias } \\
\text { anteriormente controlada com } \\
\text { risco de vida; paciente pode } \\
\text { necessitar de hospitalização } \\
\text { prolongada. }\end{array}$ & $\begin{array}{c}\text { Infiltrado pulmonar e/ou fibrose, } \\
\text { pneumonite, neuropatia } \\
\text { periférica, tremor, cefaleia, } \\
\text { vertigem, bisfadiga, insônia e } \\
\text { ataxia, fotossensibilização, } \\
\text { hipotireoidismo ou } \\
\text { hipertireoidismo, depósitos na } \\
\text { córnea, com repercussões visuais, } \\
\text { IC, bradicardia, hipotensão, } \\
\text { intolerância digestiva, anorexia, } \\
\text { náusea/vômito, obstipação, } \\
\text { aumento enzimas hepáticas. }\end{array}$ & $\begin{array}{l}\mathrm{AB}-\text { comp. } 200 \mathrm{mg} \\
\mathrm{FP}-\text { comp. } 200 \mathrm{mg}\end{array}$ \\
\hline ANLODIPINA & $\begin{array}{l}\text { Horário ideal de tomada: na parte da manhã, } \\
\text { na hora do café da manhã (há pouca } \\
\text { evidência de que haja um momento ideal para } \\
\text { tomar anlodipino uma vez ao dia; no entanto, } \\
\text { o horário ideal parece ser de manhã). Pode } \\
\text { ser tomado próximo ou durante a alimentação }\end{array}$ & $\begin{array}{c}\text { A retirada abrupta deste } \\
\text { medicamento pode precipitar } \\
\text { vasoespasmo coronariano, o que } \\
\text { pode resultar em infarto do } \\
\text { miocárdio }\end{array}$ & $\begin{array}{l}\text { Edema periférico, fadiga, } \\
\text { palpitação, dor de cabeça, } \\
\text { dispepsia, náuseas }\end{array}$ & $\mathrm{AB}-$ comp. 5 e $10 \mathrm{mg}$ \\
\hline BISOPROLOL & $\begin{array}{l}\text { Horário ideal de tomada: pela manhã, antes, } \\
\text { durante ou após o café da manhã. Os } \\
\text { comprimidos devem ser engolidos inteiros, } \\
\text { com algum líquido. Este medicamento não } \\
\text { deve ser partido ou mastigado. Se esquecer, } \\
\text { instruir paciente a tomar uma dose logo que } \\
\text { possível, mas se próxima dose em menos de } \\
8 \mathrm{~h} \text {, pule a dose. }\end{array}$ & $\begin{array}{c}\text { A retirada abrupta desse } \\
\text { medicamento pode agravar a } \\
\text { angina de peito, ou causar infarto } \\
\text { do miocárdio ou arritmias } \\
\text { ventriculares. }\end{array}$ & $\begin{array}{l}\text { Bradicardia, piora da IC, tontura } \\
\text { (cuidado uso concomitante com } \\
\text { álcool), cefaleia, broncoespasmo } \\
\text { (pacientes com asma ou DPOC), } \\
\text { hipotensão, sensação de frio ou } \\
\text { dormência nas extremidades, } \\
\text { distúrbios do sono. }\end{array}$ & $\begin{array}{l}\text { INC } \\
\text { NÃO foi encontrada informação sobre } \\
\text { incorporação ao SUS (RENAME, 2014) }\end{array}$ \\
\hline
\end{tabular}


Research, Society and Development, v. 10, n. 15, e142101522899, 2021

(CC BY 4.0) | ISSN 2525-3409 | DOI: http://dx.doi.org/10.33448/rsd-v10i15.22899

\begin{tabular}{|c|c|c|c|c|}
\hline & $\begin{array}{l}\text { Vinculando o medicamento à } \\
\text { programação do paciente }\end{array}$ & $\begin{array}{c}\text { Consequências da retirada } \\
\text { do medicamento }\end{array}$ & $\begin{array}{c}\text { Causas potenciais para } \\
\text { retirada }\end{array}$ & $\begin{array}{c}\text { Alternativa para garantir provisão do } \\
\text { MEDICAMENTO }\end{array}$ \\
\hline CAPTOPRIL & $\begin{array}{l}\text { Horário ideal de tomada: incerto (embora } \\
\text { haja algumas evidências de que a } \\
\text { administração ao deitar seja uma opção } \\
\text { efetiva para reduzir o risco cardiovascular). } \\
\text { Deve ser tomado sem alimentos (1 hora antes } \\
\text { das refeições) }\end{array}$ & $\begin{array}{c}\text { A retirada abrupta desse } \\
\text { medicamento pode precipitar o } \\
\text { rebote hipertensivo em pacientes } \\
\text { diabéticos com insuficiência renal } \\
\text { crônica. }\end{array}$ & $\begin{array}{l}\text { Tosse seca, hipercalemia } \\
\text { (especialmente se usado com } \\
\text { espironolactona; os principais } \\
\text { sintomas: palpitações e fraqueza } \\
\text { muscular), hipotensão postural }\end{array}$ & $\begin{array}{l}\text { AB - comp. } 25 \mathrm{mg} \\
\text { AFP - comp. } 25 \mathrm{mg} \\
\mathrm{FP}-\text { comp. } 25 \mathrm{mg}\end{array}$ \\
\hline CARVEDILOL & $\begin{array}{c}\text { Horário ideal de tomada: noite. } \\
\text { Pode ser tomado próximo ou durante a } \\
\text { alimentação }\end{array}$ & $\begin{array}{l}\text { Taquicardia, palpitações, } \\
\text { sudorese excessiva, dor no peito, } \\
\text { infarto, morte }\end{array}$ & $\begin{array}{c}\text { Fadiga, hipotensão, diarreia, } \\
\text { astenia, bradicardia, tonturas } \\
\text { (cuidado uso concomitante com } \\
\text { álcool), edema } \\
\end{array}$ & $\begin{array}{l}\text { AB - comp. 3,125, 6,25, } 12,5 \text { e } 25 \text { mg } \\
\text { Farmácia Ambulatorial da Instituição }\end{array}$ \\
\hline CIPROFIBRATO & Horário ideal de tomada: não encontrado. & & $\begin{array}{l}\text { Cefaleia, vertigem, tonturas, } \\
\text { sonolência, náuseas, vômitos, dor } \\
\text { abdominal, mialgia, rash, } \\
\text { alopecia, fadiga. }\end{array}$ & $\mathrm{AB}-$ comp. $100 \mathrm{mg}$ \\
\hline DABIGATRANA & $\begin{array}{l}\text { Horário ideal de tomada: não documentado. } \\
\text { Pode ser ingerido com ou sem alimentos e } \\
\text { com um copo de água. Em caso de } \\
\text { desenvolvimento de sintomas } \\
\text { gastrointestinais, é recomendado administrar } \\
\text { com alimentos e/ou com um inibidor da } \\
\text { bomba de prótons. Este medicamento não } \\
\text { deve ser aberto ou mastigado. No manuseio } \\
\text { retire a película de alumínio e remova a } \\
\text { cápsula. A cápsula não deve ser empurrada } \\
\text { através da folha de alumínio e nem } \\
\text { armazenada em qualquer outro recipiente } \\
\text { diferente do original (caixa de comprimidos } \\
\text { ou organizadores). }\end{array}$ & $\begin{array}{l}\text { A descontinuação ou interrupção } \\
\text { da terapia, sem anticoagulação } \\
\text { alternativa adequada aumenta o } \\
\text { risco de eventos trombóticos, } \\
\text { incluindo acidente vascular } \\
\text { cerebral. }\end{array}$ & $\begin{array}{c}\text { Sangramento, anemia, dor } \\
\text { abdominal, diarreia, desconforto } \\
\text { digestivo pós-refeição, náusea e } \\
\text { hematúria. }\end{array}$ & $\begin{array}{l}\text { NÃO foi encontrada informação sobre } \\
\text { incorporação ao SUS (RENAME, 2014) }\end{array}$ \\
\hline DIGOXINA & $\begin{array}{l}\text { Horário ideal de tomada: não encontrado. } \\
\text { Orientar para utilizar com estômago vazio, } \\
\text { mas se houver desconforto gástrico usar com } \\
\text { alimento. }\end{array}$ & & $\begin{array}{l}\text { Arritmias, Hipopotassemia, } \\
\text { hipomagnesemia; Náuseas, } \\
\text { vômito; }\end{array}$ & $\begin{array}{l}\mathrm{AB} \text { - comp. } 0,25 \mathrm{mg} \\
\mathrm{FP} \text { - comp. } 0,25 \mathrm{mg}\end{array}$ \\
\hline ENALAPRIL & $\begin{array}{l}\text { Horário ideal de tomada: noite. } \\
\text { Pode ser tomado próximo ou durante a } \\
\text { alimentação }\end{array}$ & $\begin{array}{c}\text { A retirada abrupta desses } \\
\text { medicamentos pode precipitar } \\
\text { rebote hipertensivo em pacientes } \\
\text { diabéticos com insuficiência renal } \\
\text { crônica }\end{array}$ & $\begin{array}{l}\text { Hipotensão, tontura, cefaleia, } \\
\text { tosse seca. O álcool aumenta seu } \\
\text { efeito hipotensor. }\end{array}$ & $\begin{array}{l}\text { AB - comp. } 5,10 \text { e } 20 \mathrm{mg} \\
\text { AFP - comp. } 10 \mathrm{mg} \\
\text { FP - comp. } 5 \text { e de } 20 \mathrm{mg}\end{array}$ \\
\hline
\end{tabular}


Research, Society and Development, v. 10, n. 15, e142101522899, 2021

(CC BY 4.0) | ISSN 2525-3409 | DOI: http://dx.doi.org/10.33448/rsd-v10i15.22899

\begin{tabular}{|c|c|c|c|c|}
\hline & $\begin{array}{l}\text { Vinculando o medicamento à } \\
\text { programação do paciente }\end{array}$ & $\begin{array}{c}\text { Consequências da retirada } \\
\text { do medicamento }\end{array}$ & $\begin{array}{c}\text { Causas potenciais para } \\
\text { retirada }\end{array}$ & $\begin{array}{c}\text { Alternativa para garantir provisão do } \\
\text { MEDICAMENTO }\end{array}$ \\
\hline $\begin{array}{l}\text { ESPIRONOLAC- } \\
\text { TONA }\end{array}$ & $\begin{array}{l}\text { Horário ideal de tomada: não documentado. } \\
\text { Pode ser tomado próximo ou durante a } \\
\text { alimentação (ideal para minimizar efeitos } \\
\text { adversos gástricos e } \uparrow \text { biodisponibilidade). } \\
\text { Este medicamento não deve ser partido, } \\
\text { aberto ou mastigado. Recomendar não ingerir } \\
\text { bebida alcoólica concomitantemente a } \\
\text { espironolactona. }\end{array}$ & $\begin{array}{l}\text { Não há efeito rebote aparente } \\
\text { após a suspensão abrupta do } \\
\text { tratamento com espironolactona }\end{array}$ & $\begin{array}{l}\text { Hipercalemia (principais } \\
\text { sintomas: palpitações e fraqueza } \\
\text { muscular), ginecomastia, } \\
\text { irregularidades menstruais. }\end{array}$ & $\mathrm{AB}-$ comp. 25 e $100 \mathrm{mg}$ \\
\hline FUROSEMIDA & $\begin{array}{c}\text { Horário ideal de tomada: não há diferença } \\
\text { significativa em relação } \\
\text { aos horários de tomada pela manhã e à noite. } \\
\text { Pode ser tomado } \\
\text { próximo ou durante a alimentação }\end{array}$ & $\begin{array}{c}\text { Se o uso de diuréticos for } \\
\text { interrompido de repente em } \\
\text { pacientes com ingestão normal de } \\
\text { sódio, haverá retenção rebote de } \\
\text { sódio e água (com consequente } \\
\text { edema), porque os mecanismos } \\
\text { compensatórios que mantêm o } \\
\text { equilíbrio de sódio na presença de } \\
\text { diuréticos continuam a agir por } \\
\text { vários dias após a diurese ter sido } \\
\text { interrompida }\end{array}$ & $\begin{array}{l}\text { Hipocalemia (os sintomas } \\
\text { incluem fraqueza muscular, } \\
\text { mialgia e cãibras musculares), } \\
\text { hiperglicemia, hiponatremia (os } \\
\text { sintomas incluem náuseas e } \\
\text { vômitos, dor de cabeça, fadiga, } \\
\text { perda de apetite) }\end{array}$ & $\begin{array}{l}\mathrm{AB} \text { - comp. } 40 \mathrm{mg} \\
\mathrm{FP} \text { - comp. } 40 \mathrm{mg}\end{array}$ \\
\hline HIDRALAZINA & $\begin{array}{l}\text { Horário ideal de tomada: não encontrado. } \\
\text { Tomar todos os dias no mesmo horário da } \\
\text { mesma forma com ou sem alimentos. Alertar } \\
\text { que pode afetar a capacidade de realizar } \\
\text { atividades que exigem atenção e coordenação } \\
\text { motora como operar máquinas e dirigir, pelo } \\
\text { risco de produzir tontura. Esse medicamento } \\
\text { não pode ser partido, aberto ou mastigado. }\end{array}$ & & $\begin{array}{c}\text { Anorexia, náusea, vômito, } \\
\text { diarreia. Cefaleia grave, } \\
\text { neuropatia periférica Indução de } \\
\text { lúpus eritematoso sistêmico. O } \\
\text { álcool aumenta seu efeito } \\
\text { hipotensor. }\end{array}$ & $\mathrm{AB}-$ comp. 25 e $50 \mathrm{mg}$ \\
\hline $\begin{array}{l}\text { HIDROCLORO- } \\
\text { TIAZIDA }\end{array}$ & $\begin{array}{c}\text { Horário ideal de tomada: antes das 18:00 } \\
\text { horas, de preferência } \\
\text { na parte da manhã. Pode ser tomado próximo } \\
\text { ou durante a } \\
\text { alimentação. Proteger a pele do sol com uso } \\
\text { de protetor solar. }\end{array}$ & & $\begin{array}{c}\text { Hipocalemia (os sintomas } \\
\text { incluem fraqueza muscular, } \\
\text { mialgia e cãibras musculares), } \\
\text { fraqueza, hipercalcemia, } \\
\text { hiponatremia, fotossensibilidade, } \\
\text { hipotensão ortostática } \\
\text { (principalmente quando associada } \\
\text { com álcool). }\end{array}$ & $\begin{array}{l}\text { AB - comp. } 12,5 \text { e } 25 \mathrm{mg} \\
\text { AFP - comp. } 25 \mathrm{mg} \\
\mathrm{FP}-\text { comp. } 25 \mathrm{mg}\end{array}$ \\
\hline
\end{tabular}


Research, Society and Development, v. 10, n. 15, e142101522899, 2021

(CC BY 4.0) | ISSN 2525-3409 | DOI: http://dx.doi.org/10.33448/rsd-v10i15.22899

\begin{tabular}{|c|c|c|c|c|}
\hline & $\begin{array}{l}\text { Vinculando o medicamento à } \\
\text { programação do paciente }\end{array}$ & $\begin{array}{c}\text { Consequências da retirada } \\
\text { do medicamento }\end{array}$ & $\begin{array}{c}\text { Causas potenciais para } \\
\text { retirada }\end{array}$ & $\begin{array}{l}\text { Alternativa para garantir provisão do } \\
\text { MEDICAMENTO }\end{array}$ \\
\hline IVABRADINA & $\begin{array}{l}\text { Horário ideal de tomada: os comprimidos } \\
\text { devem ser administrados por via oral, duas } \\
\text { vezes ao dia, isto é, uma vez pela manhã e } \\
\text { outra à noite durante as refeições. }\end{array}$ & & $\begin{array}{l}\text { Bradicardia, dor de cabeça } \\
\text { (principalmente no primeiro } \\
\text { mês), fenômenos luminosos } \\
\text { (aumento transitório da } \\
\text { luminosidade numa área limitada } \\
\text { do campo visual), visão turva, } \\
\text { fibrilação atrial e pressão } \\
\text { arterial não controlada. }\end{array}$ & NÃO incorporado ao SUS (RENAME, 2014) \\
\hline LOSARTANA & $\begin{array}{l}\text { Horário ideal de tomada: investigações ainda } \\
\text { não conclusivas. } \\
\text { Pode ser tomado com alimentos (uso } \\
\text { concomitante da losartana e do suco de } \\
\text { toranja pode resultar em aumento da meia- } \\
\text { vida e diminuição da concentração do } \\
\text { metabólito ativo) } \\
\end{array}$ & $\begin{array}{l}\text { Há poucas evidências de efeito } \\
\text { rebote ou de semelhante natureza } \\
\text { após a interrupção abrupta da } \\
\text { losartana }\end{array}$ & Tontura, diarréia, cansaço & $\begin{array}{l}\mathrm{AB}-\text { comp. } 50 \mathrm{mg} \\
\mathrm{AFP}-\text { comp. } 50 \mathrm{mg} \\
\mathrm{FP}-\text { comp. } 25 \mathrm{mg}\end{array}$ \\
\hline $\begin{array}{l}\text { MONONITRATO } \\
\text { DE } \\
\text { ISOSSORBIDA }\end{array}$ & $\begin{array}{l}\text { Horário ideal de tomada: não encontrado. } \\
\text { Orientar para ingerir o comprimido com } 250 \\
\text { mL de água. }\end{array}$ & & $\begin{array}{l}\text { Cefaléia, tontura, náuseas, vômito } \\
\text { e hipotensão ortostática } \\
\text { (principalmente quando associada } \\
\text { com álcool). }\end{array}$ & $\begin{array}{l}\mathrm{AB}-\text { comp. } 20 \mathrm{e} 40 \mathrm{mg} \\
\mathrm{FP}-\text { comp. } 20 \mathrm{mg}\end{array}$ \\
\hline SINVASTATINA & Horário ideal de tomada: à noite. & & $\begin{array}{c}\text { Miopatia, rabdomiólise, } \\
\text { hepatotoxicidade, elevação de } \\
\text { creatina cinase, dor abdominal, } \\
\text { náuseas, vômitos e diarreia, } \\
\text { distúrbios psiquiátricos, síndrome } \\
\text { das pernas inquietas, distúrbios } \\
\text { visuais. } \\
\end{array}$ & $\begin{array}{l}\text { AB - comp. 10, } 20 \text { e } 40 \mathrm{mg} \\
\text { AFP - comp. 10, } 20 \text { e } 40 \mathrm{mg} \\
\text { FP - comp. } 20 \mathrm{mg}\end{array}$ \\
\hline TRIMETAZIDINA & $\begin{array}{l}\text { Horário ideal de tomada: durante as } \\
\text { refeições. Os comprimidos devem ser } \\
\text { ingeridos com copo de água, no momento das } \\
\text { refeições (no caso da posologia } 2 \mathrm{x} / \mathrm{dia} \text {, tomar } \\
\text { durante o café da manhã e o jantar). Este } \\
\text { medicamento não deve ser partido, aberto ou } \\
\text { mastigado. }\end{array}$ & & $\begin{array}{c}\text { Tonturas, cefaleia, dor } \\
\text { abdominal, diarréia, dispepsia, } \\
\text { náuseas e vômitos erupção } \\
\text { cutânea, prurido, urticária, } \\
\text { astenia. }\end{array}$ & $\begin{array}{l}\text { Não foi encontrada informação sobre } \\
\text { incorporação ao SUS (RENAME, 2014) }\end{array}$ \\
\hline
\end{tabular}


Research, Society and Development, v. 10, n. 15, e142101522899, 2021

(CC BY 4.0) | ISSN 2525-3409 | DOI: http://dx.doi.org/10.33448/rsd-v10i15.22899

\begin{tabular}{|c|c|c|c|c|}
\hline & $\begin{array}{l}\text { Vinculando o medicamento à } \\
\text { programação do paciente }\end{array}$ & $\begin{array}{c}\text { Consequências da retirada } \\
\text { do medicamento }\end{array}$ & $\begin{array}{c}\text { Causas potenciais para } \\
\text { retirada }\end{array}$ & $\begin{array}{c}\text { Alternativa para garantir provisão do } \\
\text { MEDICAMENTO }\end{array}$ \\
\hline VARFARINA & $\begin{array}{l}\text { Horário ideal de tomada: recomendamos } \\
\text { sempre tomar o anticoagulante oral no } \\
\text { mesmo horário, de preferência no final da } \\
\text { tarde, em torno das } 17 \text { horas. Se esquecer de } \\
\text { tomar a dose deve ser tomada assim que } \\
\text { possível no mesmo dia. A ingestão de álcool } \\
\text { aumenta o efeito anticoagulante. Não se deve } \\
\text { ingerir álcool durante o tratamento. }\end{array}$ & $\begin{array}{c}\text { A descontinuação ou interrupção } \\
\text { da terapia, sem anticoagulação } \\
\text { alternativa adequada aumenta o } \\
\text { risco de eventos trombóticos, } \\
\text { incluindo acidente vascular } \\
\text { cerebral. }\end{array}$ & $\begin{array}{c}\text { Anemia, dor no peito, } \\
\text { sangramento, fadiga, mal-estar, } \\
\text { dor abdominal, inchaço, palidez, } \\
\text { mialgia, tontura, cefaleias e } \\
\text { síndrome dos dedos roxos. }\end{array}$ & $\mathrm{AB}-$ comp. 1 e $5 \mathrm{mg}$ \\
\hline
\end{tabular}

AB - Medicamento de Atenção Básica (RENAME, 2014); AFP - Aqui tem Farmácia Popular (farmácias e drogarias conveniadas); FP - Farmácia Popular (rede própria).

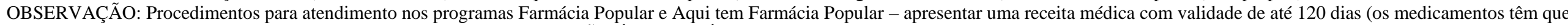
estar prescritos nas doses disponíveis) e um documento com foto e CPF. NÃO É NECESSÁRIO TER CADASTRO.

Fonte: Adaptado de Camuzi (2017). Observação: As orientações se baseiam nos procedimentos e regulamentos vigentes à época do estudo. 


\section{Agradecimentos}

À Fundação Carlos Chagas Filho de Amparo à Pesquisa do Estado do Rio de Janeiro (FAPERJ), pelo incentivo e apoio financeiro que viabilizou o início do projeto "Implementação de Ações para Otimização da Assistência Farmacêutica a Pacientes com Insuficiência Cardíaca”.

Ao Programa de Pós-Graduação Ciências Aplicadas a Produtos para a Saúde (PPG-CAPS); ao Instituto Nacional de Cardiologia (INC); ao Programa de Pós-graduação Lato Sensu Residência em Farmácia Hospitalar; ao Ministério da Saúde (MS); à Universidade Federal Fluminense (UFF).

\section{Referências}

Albuquerque, D. C., Souza Neto, J. D., Bacal, F., Rohde, L. E., Bernardez-Pereira, S., Berwanger, O., \& Almeida, D. R. (2015). I Brazilian Registry of Heart Failure - Clinical Aspects, Care Quality and Hospitalization Outcomes. Arq Bras Cardiol, 104(6), 433-442. https://doi.org/10.5935/abc.20150031

Barretto, A. C. P., del Carlo, C. H., Cardoso, J. N., Morgado, P. C., Munhoz, R. T., Eid, M. O., Oliveira Jr, M. T., Scipioni, A. R., \& Ramires, J. A. F. (2008). Re-hospitalizações e morte por insuficiência cardíaca - Índices ainda alarmantes / Hospital readmissions and death from heart failure - Rates still alarming. Arquivos Brasileiros de Cardiologia, 91(5), 309-341. https://doi.org/10.1590/S0066-782X2008001700009

Bocchi, E. A., Braga, F. G. M., Ferreira, S. M. A., Rohde, L. E. P., Oliveira, W. A. de, Almeida, D. R. de, Moreira, M. da C. V., Bestetti, R. B., Bordignon, S., Azevedo, C., Tinoco, E. M., Rocha, R. M., Issa, V. S., Ferraz, A., Cruz, F. das D., Guimarães, G. V., Montera, V. dos S. P., Albuquerque, D. C., Bacal, F., ... Montera, M. W. (2009). III Diretriz Brasileira de Insuficiência Cardíaca Crônica. Arquivos Brasileiros de Cardiologia, 93 , 3-70.

Brasil, M. da S. (2010). Formulário terapêutico nacional 2010: Rename 2010. http://www.saude.gov.br/bvs

Brasil, M. da S. (2015). Relação Nacional de Medicamentos Essenciais: RENAME 2014 (Ministério da Saúde, Ed.; 9th ed.). Ministério da Saúde. www.saude.gov.br/medicamentos

Camuzi, R. C. (2017). Adesão de pacientes com insuficiência cardíaca à farmacoterapia: as experiências de dois centros clínicos especializados. https://doi.org/10.13140/RG.2.2.28686.72007

Camuzi, R. C., Cordeiro, B. C., Elias, S. C., Martins, W. de A., Mesquita, E. T., \& Castilho, S. R. de. (2016). Perfil de utilização e adesão ao tratamento medicamentoso por pacientes atendidos em Clínica de Insuficiência Cardíaca de um Hospital Universitário da Região Metropolitana do Rio de Janeiro. Rev Bras Farm, 97(Sup), 61-77.

Camuzi, R. C., Moraes, J. R. de, Oliveira, E. B. de, Peixoto, R. T., Mesquita, E. T., \& Castilho, S. R. de. (2021). Adesão farmacoterapêutica em insuficiência cardíaca: estudo exploratório num centro especializado fluminense. Brazilian Journal of Health Review, 4(6). No prelo.

CNPq. (2021). Glossário - $\quad$ Plataforma Clossário. http://lattes.cnpq.br/web/dgp/glossario?p_p_id=54_INSTANCE_QoMcDQ9EVoSc\&_54_INSTANCE_QoMcDQ9EVoSc_struts_action=\%2Fwiki_display\%2 Fview\&_54_INSTANCE_QoMcDQ9EVoSc_nodeName=Main\&_54_INSTANCE_QoMcDQ9EVoSc_title=Pesquisa

Culig, J., Leppee, M., Boskovic, J., \& Eric, M. (2011). Determining the difference in medication compliance between the general patient population and patients receiving antihypertensive therapy: a case study. Archives of Pharmacal Research, 34(7), 1143-1152. https://doi.org/10.1007/s12272-011-0712-0

Davis, E. M., Packard, K. A., \& Jackevicius, C. A. (2014). The Pharmacist Role in Predicting and Improving Medication Adherence in Heart Failure Patients. Journal of Managed Care Pharmacy, 20(7), 741-755. https://doi.org/10.18553/jmcp.2014.20.7.741

Despaigne, R. M., Sanchez, C. M. D., \& Sarfraz, A. (2012). Therapeutic adherence in outpatients with heart failure registered with a community pharmacy. Braz. J. Pharm. Sci., 48(1), 61-68. http://dx.doi.org/10.1590/S1984-82502012000100007

Fitzgerald, A. A., Powers, J. D., Ho, P., Maddox, T. M., Peterson, P., Allen, L., Masoudi, F. A., Magid, D., \& Havranek, E. (2011). Impact of Medication Nonadherence on Hospitalizations and Mortality in Heart Failure. J. Card. Fail., 17(8), 664-669. https://doi.org/10.1016/j.cardfail.2011.04.011

Horne, R., Weinman, J., \& Hankins, M. (1999). The beliefs about medicines questionnaire: The development and evaluation of a new method for assessing the cognitive representation of medication. Psychology \& Health, 14(1), 1-24.

Jackevicius, C. A. a b d f, Li, P. e, \& Tu, J. V. b c e. (2008). Prevalence, predictors, and outcomes of primary nonadherence after acute myocardial infarction. Circulation, 117(8), 1028-1036. https://doi.org/10.1161/CIRCULATIONAHA.107.706820

Jessup, M. (2014). The Heart Failure Paradox: An Epidemic of Scientific Success: Presidential Address at the American Heart Association 2013 Scientific Sessions. Circulation, 129(25), 2717-2722. https://doi.org/10.1161/CIR.0000000000000065

Karve, S., Cleves, M. A., Helm, M., Hudson, T. J., West, D. S., \& Martin, B. C. (2009). Prospective validation of eight different adherence measures for use with administrative claims data among patients with schizophrenia. Value in Health: The Journal of the International Society for Pharmacoeconomics and Outcomes Research, 12(6), 989-995. https://doi.org/10.1111/j.1524-4733.2009.00543.x

López Cabezas, C. a e f, Falces Salvador, C. b g, Cubí Quadrada, D. a f, Arnau Bartés, A. c h, Ylla Boré, M. a f, Muro Perea, N. d i, \& Homs Peipoch, E. a f. (2006). Randomized clinical trial of a postdischarge pharmaceutical care program vs. regular follow-up in patients with heart failure [Ensayo clínico aleatorizado 
Research, Society and Development, v. 10, n. 15, e142101522899, 2021 (CC BY 4.0) | ISSN 2525-3409 | DOI: http://dx.doi.org/10.33448/rsd-v10i15.22899

de un programa de atención farmacéutica al alta frente a seguimiento habitual en pacientes con ins. Farmacia Hospitalaria, 30(6), 328-342. https://doi.org/10.1016/S1130-6343(06)74004-1

Lowry, K. P., Dudley, T. K., Oddone, E. Z., \& Bosworth, H. B. (2005). Intentional and unintentional nonadherence to antihypertensive medication. The Annals of Pharmacotherapy, 39(7-8), 1198-1203. https://doi.org/10.1345/aph.1E594

Mangini, S., Silveira, F. S., Silva, C. P., Grativvol, P. S., Seguro, L. F. B. da C., Ferreira, S. M. A., Mocelin, A. O., Cardoso, L. F., Bacal, F., \& Bocchi, E. A. (2008). Decompensated heart failure in the emergency department of a cardiology hospital. Arquivos Brasileiros de Cardiologia, 90(6), 433-440.

Marinker, M., \& Shaw, J. (2003). Not to be taken as directed. BMJ, 326(7385), 348-349.

Morisky, D. E., Green, L. W., \& Levine, D. M. (1986). Concurrent and predictive validity of a self-reported measure of medication adherence. Medical Care, 24(1), 67-74. http://www.ncbi.nlm.nih.gov/pubmed/3945130

Morley, P. C., Strand, L. M., \& Cipolle, R. J. (2004). Pharmaceutical Care Practice: The Clinician's Guide.

Náfrádi, L., Galimberti, E., Nakamoto, K., \& Schulz, P. J. (2016). Intentional and Unintentional Medication Non-Adherence in Hypertension: The Role of Health Literacy, Empowerment and Medication Beliefs. Journal of Public Health Research, 5(3), 762. https://doi.org/10.4081/jphr.2016.762

Nieuwlaat, R., Wilczynski, N., Navarro, T., Hobson, N., Jeffery, R., Keepanasseril, A., Agoritsas, T., Mistry, N., Iorio, A., Jack, S., Sivaramalingam, B., Iserman, E., Mustafa, R. A., Jedraszewski, D., Cotoi, C., \& Haynes, R. B. (2014). Interventions for enhancing medication adherence. Cochrane Database Syst Rev, 11, CD000011. https://doi.org/10.1002/14651858.CD000011.pub4

Oliveira-Filho, A. D., Morisky, D. E., Costa, F. A., Pacheco, S. T., Neves, S. F., \& Lyra-Jr, D. P. (2014). Improving post-discharge medication adherence in patients with CVD: a pilot randomized trial. Arq Bras Cardiol, 103(6), 503-512. https://doi.org/10.5935/abc.20140151

Roger, V. L. (2013). Epidemiology of Heart Failure. Circulation Research, 113(6), 646-659.

Rohde, L. E. P., Montera, M. W., Bocchi, E. A., Clausell, N. O., Albuquerque, D. C. de, Rassi, S., Colafranceschi, A. S., Freitas Junior, A. F. de, Ferraz, A. S., Biolo, A., Barretto, A. C. P., Ribeiro, A. L. P., Polanczyk, C. A., Gualandro, D. M., Almeida, D. R., Silva, E. R. R. da, Figueiredo, E. L., Mesquita, E. T., Marcondes-Braga, F. G., ... Martins, W. de A. (2018). Diretriz Brasileira de Insuficiência Cardíaca Crônica e Aguda. Arquivos brasileiros de cardiologia, $111(3), 436-539$

van der Wal, M. H. L., \& Jaarsma, T. (2008). Adherence in heart failure in the elderly: Problem and possible solutions. International Journal of Cardiology, 125(2), 203-208. https://doi.org/10.1016/j.ijcard.2007.10.011

WHO, W. H. O. (2003). Adherence to long-term therapies: http://www.who.int/chp/knowledge/publications/adherence_introduction.pdf

evidence for action. World Health Organization. 